\title{
EFECTO TRIBUTARIO DE LAS DONACIONES EN EL IMPUESTO \\ SOBRE LA RENTA Y COMPLEMENTARIOS
}

\section{Tax effect of donations on income tax and complementary taxes}

\author{
Hernán José Hernández-Belaides ${ }^{1}$
}

Recibido: Marzo 22 de 2016/Aceptado: Agosto 4 de 2016

\begin{abstract}
RESUMEN
Esta investigación tiene como objetivo dilucidar las incidencias tributarias y el efecto que tienen las donaciones en el impuesto sobre la renta y complementarios de los contribuyentes que hacen la donación. Es común encontrar una serie de opiniones al respecto que riñen con las normas tributarias o que simplemente no tienen un real asidero legal, lo cual se manifiesta en la desinformación de aquellas personas altruistas que buscan por este medio contribuir con causas nobles y de interés, generalmente, de poblaciones vulnerables. Se analizan las leyes y normas relacionadas y dentro de un contexto legal y práctico, se harán las precisiones tendientes al cumplimiento de los objetivos.
\end{abstract}

Palabras clave: Donaciones, Lucro, Altruismo, Filantropía, Solidaridad, Impuestos.

\section{ABSTRACT}

The purpose of this research is to elucidate the tax incidence and the effect that donations have on the income tax and complementary taxpayers who make the donation. It is common to find a series of opinions in this regard that conflict with the tax rules or that simply do not have a real legal basis, which manifests itself in the misinformation of those altruistic people who seek to contribute with noble and interesting causes, generally, of vulnerable populations. The related laws and norms are analyzed and within a legal and practical context, the precisions are made tending to the fulfillment of the objectives.

Keywords: Donations, Profit, Altruism, Philanthropy, Solidarity, Taxes.

Cómo referenciar este artículo: Hernández, H. (2016). Efecto tributario de las donaciones en el impuesto sobre la renta y complementarios. Ad-Gnosis, 5(5), 37-46.

1. Contador Público, Universidad del Atlántico, Colombia. Especialista en Tributación, Universidad del Norte, Colombia. Maestrante en Fiscalidad Internacional de la Universidad Internacional de La Rioja UNIR. hernanhdezb@gmail.com ORCID code 0000-0002-0152-7301 


\section{Introducción}

El propósito de esta investigación es poner de presente las implicaciones tributarias y el efecto que causan las donaciones en el impuesto sobre la renta y complementarios en Colombia. Muchas entidades sin fines de lucro subsisten con las donaciones que reciben de empresas y de personas físicas altruistas, que contribuyen para que esas entidades cumplan, generalmente con una labor social, dirigida a personas o grupos de personas, que tienen una situación económica desfavorable o se encuentran en estado vulnerable.

Veremos las regulaciones legales y normativas sobre las cuales navegan este tipo de entidades poniendo de presente el impacto que generan en la sociedad a través de beneficios sociales individuales y colectivos. Está dirigido a estudiantes de ciencias económicas y jurídicas y a los profesionales que se desempeñan en estas áreas del conocimiento, además, de todos aquellos interesados en conocer el origen, los procesos y la normativa regulatoria de las entidades sin fines de lucro y también, del proceso y los beneficios tributarios que implican las donaciones.

\section{Metodología}

El método de investigación utilizado es el deductivo, es decir, se analizan leyes y normas establecidas en relación con las entidades sin fines de lucro y el marco legal de las donaciones. Este documento contiene una breve historia de entidades sin fines de lucro y la descripción de las mismas enmarcadas en leyes regulativas y un análisis del marco regulativo de las donaciones y de la incidencia que tienen en el impuesto de renta en Colombia.

\section{Lucro}

El lucro puede describirse como el deseo de obtener o generar ganancias relacionadas con cualquier actividad, para quienes las realizan, a favor de socios o inversionistas. Generalmente, las personas o sociedades buscan obtener, mediante una actividad, comercial o no, un beneficio económico o una utilidad por su realización.

Sin embargo, existen personas y entidades que rompen este paradigma y se dedican a la realización de actividades sin contraprestación económica, es decir, sin fines de lucro. Buscan generar mejor calidad de vida para sectores vulnerables o menos favorecidos a través de programas de caridad que llevan a estas comunidades salud, alimentación, recreación, entre otros.

Estas acciones, loables por demás, tienen su origen en los albores de la humanidad, con influencia generalmente religiosa, enmarcadas en la caridad. El imperio romano, cuando entra en contacto con las tradiciones judías del diezmo, que estaba dirigido a ayudar a los más necesitados, realiza actividades tendientes a beneficiar a la población vulnerable.

\section{Filantropía}

Flavio Claudio Juliano, último emperador pagano del Imperio Romano, desde el año 361 
hasta su muerte a manos de los cristianos el 26 de junio del 363, introduce el término filantropía con la finalidad única de reemplazar el término cristiano caridad, que era una virtud de esa nueva religión.

En la antigua Grecia la filantropía tenía como significado primordial el "amor por el ser humano" o "amor a la humanidad". La definición griega ha prevalecido en el tiempo, entendido esto como un sentimiento humanitario que mueve a las personas a hacer el bien sin esperar contraprestación alguna. De ahí tomó su significado la Real Academia de la Lengua Española, RAE (2014), que la define como: "Amor al género humano".

La filantropía se trata, según Rey García (2013) "de una institución que no solo es sorprendentemente prevalente a lo largo de distintas etapas históricas y a lo ancho de diferentes marcos culturales, sino que además ha mutado de modo sustantivo en sus manifestaciones y percepciones a través de todos ellos".

Una mutación o evolución notable de la filantropía en los años más recientes, tiene que ver con nuevos matices que se están generando a nivel global. Un nuevo término se abre paso con mucha fuerza, se trata del filantrocapitalismo descrito por Bishop y Green (2009), citados por Manzano (2013) como una nueva forma de hacer filantropía siguiendo los criterios por los que se mueve el mundo de los negocios en el capitalismo.
Este fenómeno, en palabras de Rey García (2013):

tiene mucho que ver con la irrupción de un grupo de empresarios de éxito, procedentes muchos de ellos del sector de las nuevas tecnologías, que han alcanzado elevadísimas cotas de riqueza siendo aún muy jóvenes y que creen que pueden aplicar su experiencia a hacer el bien. La diversidad de sus procedencias sociales, su deseo de contribuir a paliar algunos de los problemas del mundo y su voluntad de involucrarse directamente en dichas tareas -más allá de su mera aportación económica- han dado lugar a esta nueva generación de filántropos.

La filantropía vista de este modo, nos pone a reflexionar sobre la recompensa de quienes entregan parte de sus fortunas en favor de otros seres humanos con menos oportunidades o menos favorecidos. Es adecuada una reflexión en este sentido, hecha por Sáenz de Miera (2003), en los siguientes términos:

... Había acaparado, como suelen hacer en sus puestos los hombres industriosos, una parte mayoritaria en la administración de las instituciones benéficas de la ciudad y sus caridades privadas eran tanto diminutas como abundantes (...) Esta es la forma en la que un hombre va acumulando poder, sobre las esperanzas y los temores de sus vecinos, 
así como la gratitud de estos, y el poder, una vez se infiltra en esa sutil región, se propaga, extendiéndose fuera de toda proporción a sus medios externos.

\section{Entidades Sin Ánimo de Lucro}

La financiación del desarrollo ha cambiado notablemente y ahora, la tarea que le corresponde al Estado, está siendo tomada por personas de buen corazón. Esas personas, filántropos, altruistas generosos y benévolos, con el ánimo de ejecutar de mejor manera su tarea beneficiaria, crearon unas entidades, personas jurídicas independientes, con la finalidad o con el objeto de prestar o suplir necesidades, clasificadas estas según Simancas (2012), quién cita a Keith y Newstrom (2002) en necesidades primarias y secundarias, siendo las primarias la carencia de alimento y agua por ser vitales para preservar la vida.

Estas entidades, cobijadas generalmente por una legislación especial, tienen distintas denominaciones según el área geográfica donde se desarrollen, pero todas conservan la misma filosofía basada en el servicio desinteresado, por los más necesitados. En Colombia están denominadas como Entidades Sin Ánimo de Lucro (ESAL), pero en otros países son nombradas como entidades sin fines de lucro, entidades no lucrativas, organizaciones sin fines de lucro, etc.

Las organizaciones sin ánimo de lucro pueden tomar distintas figuras jurídicas, dependiendo del fin social que se propongan desarrollar.
Pueden ser fundaciones, asociaciones, corporaciones, cooperativas, clubes deportivos, academias, entre otras. Su financiamiento está dado generalmente, por las donaciones que reciben cuya destinación está dirigida a servicios solidarios y de interés para sectores específicos con necesidades especiales.

En Colombia las ESAL son personas jurídicas de derecho privado, es decir, su origen jurídico se da por la voluntad de los particulares. Aunque, como se describe en el principio de este escrito, el origen histórico se dio en el viejo continente, en el caso colombiano la historia se remonta a la época colonial. En este lapso era la iglesia católica la que pregonaba la caridad mediante la ayuda a grupos de indígenas o esclavos, a quienes se les vulneraban sus derechos fundamentales.

Años más tarde, con ocasión de la migración de los habitantes de zonas rurales hacia las cabeceras municipales o centros urbanos, impulsada y acelerada por condiciones de violencia generalizada y por situaciones políticas y sociales conflictivas que provocaron entornos de pobreza en la población, aumentando las necesidades básicas que el Estado fue incapaz de cubrir, surgieron las ESAL como iniciativa privada las cuales se encargaban de prestar un sinnúmero de servicios para paliar las insuficiencias básicas en salud, educación y servicios públicos (Ospina, 2013).

\section{Fundaciones}

La forma jurídica más utilizada para prestar 
servicios a las comunidades son las Fundaciones las cuales tienen por objeto una finalidad altruista que contribuye con el interés general. La Corte Suprema de Justicia (1940), hace las siguientes precisiones al respecto:

Las personas jurídicas regladas por el $\mathrm{C}$. C. son de dos especies: corporaciones y fundaciones de beneficencia pública. La corporación está formada por una reunión de individuos y tiene por objeto el bienestar de los asociados ya sea físico, intelectual y moral. No persigue fines de lucro. La fundación se distingue de la corporación en que es un establecimiento que persigue un fin especial de beneficencia o de educación pública, para lo cual se destinan bienes determinados. En la fundación no hay personas asociadas sino un conjunto de bienes dotados de personalidad jurídica. Las personas que por ella actúan son secundarias en contraste con las que actúan en la corporación. En la corporación hay asociación de personas, en la fundación, predestinación de bienes a fines sociales. (p.195)

En una nueva acotación al respecto, la Corte Suprema de Justicia (1947) deja claro que las fundaciones siempre serán de carácter privado, refiriéndose a este tema en los siguientes términos:

Puede un poder del Estado crear un hospital, un asilo, un instituto de artes, un colegio, etc. Un establecimiento así formado no podrá ofrecerse con los caracteres jurídicos de una Fundación, porque el establecimiento pertenecerá al Estado, quien puede gobernarlo a su arbitrio, transformarlo, extinguirlo, etc.; en tanto que la Fundación no pertenece sino a ella misma y el Estado, mientras la reconozca, no le puede cambiar sus fines lícitos.

Cabe destacar que las fundaciones son las entidades sin ánimo de lucro, que, por su condición jurídica, están relacionadas con el servicio a la comunidad o de beneficencia en general, puesto que es la asignación de un capital privado con un fin determinado, mientras que las corporaciones, a pesar de que estén constituidas sin fines de lucro, su finalidad es el resultado de una decisión o representación de personas que transforman sus opiniones personales en actos colectivos.

Continúa diciendo la Corte Suprema de Justicia (1947):

En lenguaje jurídico la palabra FUNDACIÓN representa hoy 'la constitución de cierto patrimonio con el objeto de dedicar el capital, renta, o ambas cosas conjuntamente, al cumplimiento de fines lícitos y permitidos por la ley, con carácter permanente'. Las fundaciones nacen de un acto de voluntad individual, aislado o asociado a otras voluntades, acto que traduce en obra un fin social, como 
la educación, el cultivo de las ciencias y de las artes, la caridad, etc. Toda Fundación por su naturaleza jurídica debe tener como impulso creador el de voluntades privadas. (p.622)

\section{Marco legal de las Fundaciones en Colombia}

Están descritas en el Código Civil colombiano, (Senado de la República de Colombia, 1887) en sus artículos 633 y 634, como personas jurídicas, entendiéndose estas como personas ficticias, capaces de ejercer derechos y contraer obligaciones civiles y de ser representadas judicial y extrajudicialmente, siempre que se establezcan en virtud de una ley.

El artículo 40 del Decreto 2150 de 1995 (Presidencia de la República de Colombia, 1995), autoriza la inscripción de las entidades sin ánimo de lucro directamente en las Cámaras de Comercio de su domicilio mediante documento privado. Antes de este Decreto, el cual simplificó múltiples trámites ante las entidades gubernamentales, la personería jurídica de las entidades sin ánimo de lucro se adquiría, de acuerdo al Decreto 1529 de 1990 (Presidencia de la República de Colombia, 1990) ante las gobernaciones departamentales.

\section{Régimen tributario de las fundaciones}

Tributariamente, las fundaciones están cobijadas por el Régimen tributario especial, regladas en el Artículo 19 del Estatuto Tributario (Congreso de la República de Colombia, 1989) las cuales deben cumplir una serie de requisitos para ser contribuyentes en este régimen.
Las condiciones básicas son tres y están descritas en el inciso uno de este artículo de la siguiente manera:

a) Que el objeto social principal y recursos estén destinados a actividades de salud, deporte, educación formal, cultural, investigación científica o tecnológica, ecológica, protección ambiental, o a programas de desarrollo social;

b) Que dichas actividades sean de interés general, y

c) Que sus excedentes sean reinvertidos totalmente en la actividad de su objeto social.

Debido a esta regulación y como la filosofía de las fundaciones está basada en principios filantrópicos, estas cumplen con sus fines altruistas gracias a los aportes generosos y desinteresados de personas o entidades que encuentran en la ayuda a los demás, su mayor satisfacción.

\section{Tratamiento tributario de las donaciones}

Quien dona, aparte de la satisfacción espiritual que significa ayudar a los más necesitados, también trae consigo unos beneficios económicos. Estos beneficios se verán reflejados en la disminución del impuesto sobre la renta del contribuyente filántropo que contribuye con causas nobles.

El Estatuto Tributario (Congreso de la República de Colombia, 1989) en el artículo 125 regula las donaciones y sus incidencias tanto para el donante como para el donatario. Dice el mencionado estatuto que los contribuyentes tie- 
nen derecho a deducir del impuesto de renta y complementarios el valor de las donaciones efectuadas en el periodo gravable respectivo, a las entidades sin ánimo de lucro, entre ellas las fundaciones,

cuyo objeto social y actividad correspondan al desarrollo de la salud, la educación, la cultura, la religión, el deporte, la investigación científica y tecnológica, la ecología y protección ambiental, la defensa, protección y promoción de los derechos humanos y el acceso a la justicia o de programas de desarrollo social, siempre y cuando las mismas sean de interés general.

También le pone límites al valor que puede descontarse el contribuyente de su impuesto de renta en los siguientes términos:

El valor a deducir por este concepto, en ningún caso podrá ser superior al $30 \%$ de la renta líquida del contribuyente, determinada antes de restar el valor de la donación. Esta limitación no será aplicable en el caso de las donaciones que se efectúen a los fondos mixtos de promoción de la cultura, el deporte y las artes que se creen en los niveles departamental, municipal y distrital, al Instituto Colombiano de Bienestar Familiar -ICBFpara el cumplimiento de sus programas del servicio al menor y a la familia, ni en el caso de las donaciones a las instituciones de educación superior, centros de investigación y de altos estudios para financiar programas de investigación en innovaciones científicas, tecnológicas, de ciencias sociales y mejoramiento de la productividad, previa aprobación de estos programas por el Consejo Nacional de Ciencia y Tecnología.

Para que sea viable la deducción en el impuesto de renta del donante, es necesario que la entidad que recibe la donación o entidad donataria, cumpla con unos requisitos mínimos los cuales están descritos en el artículo 125-1 del Estatuto Tributario (Congreso de la República de Colombia, 1989) de la siguiente forma:

1. Haber sido reconocida como persona jurídica sin ánimo de lucro y estar sometida en su funcionamiento a vigilancia oficial.

2. Haber cumplido con la obligación de presentar la declaración de ingresos y patrimonio o de renta, según el caso, por el año inmediatamente anterior al de la donación.

3. Manejar, en depósitos o inversiones en establecimientos financieros autorizados, los ingresos por donaciones.

Por tal motivo, el donante que tenga en mente reducir su impuesto de renta por vía de las donaciones, debe cerciorarse que la fundación cumple con los requisitos antes mencionados. En el artículo 125-2 se menciona que las donaciones pueden ser de varias modalidades, es 
decir, pueden ser en dinero, títulos valores $u$ otros activos, al respecto señala:

1. Cuando se done dinero, el pago debe haberse realizado por medio de cheque, tarjeta de crédito o a través de un intermediario financiero.

2. Cuando se donen títulos valores, se estimarán a precios de mercado de acuerdo con el procedimiento establecido por la Superintendencia de Valores.

3. Cuando se donen otros activos, su valor se estimará por el costo de adquisición más los ajustes por inflación efectuados hasta la fecha de la donación, menos las depreciaciones acumuladas hasta esa misma fecha.

En cuanto a los requisitos para reconocer la deducción por la donación, el artículo 125-3 señala que es indispensable que la entidad que recibe la donación o entidad donataria, certifique el recibo de la misma, indicando la forma, el monto y la destinación. Dicha certificación debe estar firmada por el Revisor Fiscal.

Para que el lector no saque conclusiones y para que el posible donante adquiera claridad en ese sentido, mostraré la mecánica de las donaciones en la liquidación del impuesto sobre la renta y complementarios, mediante un ejercicio práctico.

Supongamos que la empresa Accounting Re- search Ltda. presenta un estado de resultados con las siguientes características, siguiendo las reglas del artículo 26 del Estatuto Tributario:

Tabla 1. Determinación del impuesto de renta, régimen ordinario (contable)

\begin{tabular}{|l|l|c|}
\hline \multicolumn{1}{|c|}{ Detalle } & & Total \\
\hline Ingresos & & 500.000 .000 \\
\hline $\begin{array}{l}\text { Devoluciones, rebajas } \\
\text { y descuentos }\end{array}$ & & -50.000 .000 \\
\hline Ingresos netos & & 450.000 .000 \\
\hline Costos & & 280.000 .000 \\
\hline Renta bruta & & 170.000 .000 \\
\hline Deducciones & 95.000 .000 & 125.000 .000 \\
\hline Gastos deducibles & 30.000 .000 & \\
\hline $\begin{array}{l}\text { Gasto por la donación } \\
\text { efectuada }\end{array}$ & & 45.000 .000 \\
\hline $\begin{array}{l}\text { Renta líquida } \\
\text { (utilidad contable) }\end{array}$ & & \\
\hline
\end{tabular}

Fuente: Elaboración propia

De acuerdo a lo anterior la empresa tendría una utilidad contable antes de impuestos de 45 millones de pesos y su impuesto, liquidado a la tarifa del $25 \%$ vigente en el año 2015 , sería como sigue:

Tabla 2. Liquidación del impuesto de renta

\begin{tabular}{|l|c|}
\hline Utilidad Contable & 45.000 .000 \\
\hline Impuesto de Renta (Tarifa 25\%) & 11.250 .000 \\
\hline Renta Neta (Contable) & 33.750 .000 \\
\hline
\end{tabular}

Fuente: Elaboración propia

La utilidad contable corresponde a la realidad económica de la empresa. Esta utilidad es la que queda disponible para ser repartida entre los socios. Sin embargo, al momento de presentar la declaración de renta respectiva, se debe aplicar lo que al respecto anuncia la nor- 
ma tributaria en relación con las donaciones, quedando de la siguiente manera:

Tabla 3. Determinación del impuesto de renta, régimen ordinario

\begin{tabular}{|l|l|c|}
\hline \multicolumn{1}{|c|}{ Detalle } & & Total \\
\hline Ingresos & & 500.000 .000 \\
\hline $\begin{array}{l}\text { Devoluciones, rebajas } \\
\text { y descuentos }\end{array}$ & & -50.000 .000 \\
\hline Ingresos netos & & 450.000 .000 \\
\hline Costos & & 280.000 .000 \\
\hline Renta bruta & & 170.000 .000 \\
\hline Deducciones & 95.000 .000 & 95.000 .000 \\
\hline Gastos deducibles & & 75.000 .000 \\
\hline $\begin{array}{l}\text { Renta liquida antes de } \\
\text { restar la donación }\end{array}$ & & \\
\hline Fuente: Elaboración propia & &
\end{tabular}

Para aplicar correctamente la deducción fiscal por la donación, tomamos el artículo 125 del Estatuto Tributario, cuyo texto dice que el valor a deducir por este concepto, en ningún caso podrá ser superior al $30 \%$ de la renta líquida del contribuyente, determinada antes de restar el valor de la donación. En esos términos el valor máximo deducible sería:

Tabla 4. Aplicación fiscal de la donación

\begin{tabular}{|l|l|}
\hline Utilidad antes de Restar la Donación & 75.000 .000 \\
\hline Valor Máximo Deducible $(75.000 .000 \times 30 \%)$ & 22.500 .000 \\
\hline Renta Líquida Gravable & 52.500 .000 \\
\hline Impuesto de Renta (Tarifa $25 \%)$ & 13.125 .000 \\
\hline Renta Neta Fiscal & 39.375 .000 \\
\hline
\end{tabular}

Fuente: Elaboración propia

Con la aplicación de la norma tributaria aumenta significativamente el impuesto de renta, puesto que la renta líquida gravable es superior a la renta líquida contable. A simple vista pareciera que de los 30 millones de pesos que la empresa donó, estaría recuperando 22 millones 500 mil pesos, sin embargo, lo que realmente recupera por esta vía son:

Tabla 5. Recuperación de la donación en la liquidación del impuesto de renta

\begin{tabular}{|l|r|}
\hline Valor de la Donación & 30.000 .000 \\
\hline Valor Máximo Deducible (75.000.000 X $30 \%)$ & 22.500 .000 \\
\hline $\begin{array}{l}\text { Valor Recuperado a Traves del Impuesto de } \\
\text { Renta (22.500.000 X 25\%) }\end{array}$ & 5.625 .000 \\
\hline
\end{tabular}

Fuente: Elaboración propia

Nótese que la utilidad neta contable es menor que la utilidad neta fiscal, debido a la aplicación de la deducción por donación, aplicadas las normas fiscales. De esta manera se genera un mayor valor en el impuesto a pagar, esto se puede dilucidar en la siguiente tabla comparativa

Tabla 6. Comparativo del efecto de la donación entre lo contable y lo fiscal

\begin{tabular}{|l|c|c|}
\hline \multicolumn{1}{|c|}{ Detalle } & Contable & Fiscal \\
\hline $\begin{array}{l}\text { Renta Líquida antes de } \\
\text { restar la donación }\end{array}$ & 75.000 .000 & 75.000 .000 \\
\hline Menos: gasto por donación & 30.000 .000 & 22.500 .000 \\
\hline Renta Líquida Gravable & 45.000 .000 & 52.500 .000 \\
\hline Impuesto de Renta (Tarifa 25\%) & 11.250 .000 & 13.125 .000 \\
\hline
\end{tabular}

Luego de estas aclaraciones, la empresa Accounting Research Ltda. tiene claro que, de la donación efectuada por valor de 30 millones de pesos, solo podrá solicitar como deducción la suma de 22 millones 500 mil pesos lo cual le genera una disminución del impuesto de renta por valor de cinco millones seiscientos veinticinco mil pesos. 


\section{Conclusión}

La incidencia real o efectiva en el impuesto de renta está condicionada por la norma tributaria. No es cierto que la donación se pueda recuperar al $100 \%$ por la vía del impuesto de renta, pues a esta se le da el tratamiento igual que a un gasto deducible, es decir, su incidencia en el impuesto será, como máximo, el porcentaje correspondiente a la tarifa vigente en el periodo gravable en que se efectúe la donación. Diferente fuera que la donación se aplicara directamente al impuesto ya liquidado. De esa manera la recuperación sería del $100 \%$, es decir, el Estado mediante la no percepción del tributo, estaría financiando las obras sociales y de beneficencia de las entidades sin ánimo de lucro perdiéndose así, la filosofía de la filantropía que implica la ayuda de particulares a los sectores más necesitados y vulnerables.

\section{Referencias}

Congreso de la República de Colombia. Estatuto Tributario, Decreto 624 de 1989, $1989 \S(1989)$.

Corte Suprema de Justicia (1940). Gaceta Judicial. Tomo L. Nos. 1961, 1962 y 1963, p. 195.

Corte Suprema de Justicia (1947). Gaceta Judicial, Tomo LXI. Nos. 2042, 2043 y 2044, pp. 622, 622. Retrieved from http://www.organojudicial.gob.pa/ wp-content/blogs.dir/8/files/2009/libros/R.J.200110.pdf

Manzano, C. (2013). Innovación y nuevas tendencias en el ámbito de la filantropía, 19-27.
Ospina Echavarria, C. (2013). Ética Privada y la Responsabilidad Social Empresarial en las organizaciones financieras de la ciudad de Medellín. Ad-Gnosis, 2(2), 225240.

Presidencia de la República de Colombia (1990). Decreto 1529 de 1990. Diario Oficial No. 39.465, de 13 de julio de 1990.

Presidencia de la República de Colombia (1995). Decreto 2150 de 1995. Diario Oficial No. 42.137, del 6 de diciembre de 1995.

Rey García, M. (2013). Filantropía y participación cívica en el albor del siglo XXI. Participación Cívica y Filantropía, (872), 7-18.

Sáenz de Miera, A. (2003). La filantropía de Middlemarch: el poder de la generosidad. Revista Cuenta y Razón, (109), 101-105. Senado de la República de Colombia (1887). Código Civil Colombiano, (45), 1-618.

Simancas, T. R. (2012). El Marketing interno y la Calidad de Servicio de los Call Center en las instituciones bancarias de Barranquilla. Revista Ad Gnosis, 1(1), 15-37. 